\title{
Unruptured Internal Carotid-Posterior Communicating Artery Aneurysm Splitting the Oculomotor Nerve: A Case Report and Literature Review
}

\author{
Shingo Toyota ${ }^{1}$ Takuyu Taki ${ }^{1}$ Akatsuki Wakayama ${ }^{2}$ \\ ${ }^{1}$ Department of Neurosurgery, Kansai Rosai Hospital, Hyogo, Japan \\ 2 Department of Neurosurgery, Osaka Neurological Institute, \\ Osaka, Japan \\ ${ }^{3}$ Department of Neurosurgery, Osaka University Medical School, \\ Osaka, Japan
}

J Neurol Surg Rep 2014;75:e180-e182.

\begin{abstract}
Address for correspondence Shingo Toyota, MD, PhD, Department of Neurosurgery, Kansai Rosai Hospital, 3-1-69 Inabaso, Amagaski, Hyogo, 660-8511, Japan (e-mail: stshto@aol.com).
\end{abstract}

\begin{abstract}
Keywords

- fenestration

- oculomotor nerve

- internal carotidposterior communicating artery aneurysm

- three-dimensional digital subtraction angiography

Objective To report a rare case of unruptured internal carotid-posterior communicating artery (IC-PC) aneurysm splitting the oculomotor nerve treated by clipping and to review the previously published cases.

Case Presentation A 42-year-old man suddenly presented with left oculomotor paresis. Three-dimensional digital subtraction angiography (3D DSA) demonstrated a left IC-PC aneurysm with a bulging part. During surgery, it was confirmed that the bulging part split the oculomotor nerve. After the fenestrated oculomotor nerve was dissected from the bulging part with a careful microsurgical technique, neck clipping was performed. After the operation, the symptoms of oculomotor nerve paresis disappeared within 2 weeks.

Conclusions We must keep in mind the possibility of an anomaly of the oculomotor nerve, including fenestration, and careful observation and manipulation should be performed to preserve the nerve function during surgery, even though it is very rare.
\end{abstract}

\section{Introduction}

We report a rare case of unruptured internal carotid-posterior communicating artery (IC-PC) aneurysm splitting the oculomotor nerve treated by clipping and review the previously published cases.

\section{Patient}

A 42-year-old man noted sudden diplopia 2 weeks before visiting our hospital. He presented with left lid ptosis, dysfunction of the adduction of the left eyeball, and left pupil dilation. His anamnesis was not specific. Cerebral magnetic resonance angiography revealed a left IC-PC aneurysm. Vol- ume rendering images of three-dimensional digital subtraction angiography (3D DSA) demonstrated that the aneurysm, with a longer axis of $\sim 10 \mathrm{~mm}$, projected in an inferiorposterior direction and the posterior communicating artery originated from the aneurysm neck. A bulging part was noted on the surface of the aneurysm dome (-Fig. 1).

We decided to clip the aneurysm because an impending rupture was suspected. Under general anesthesia, a left frontotemporal craniotomy was performed, and the aneurysm was accessed via a pterional approach. After identification of the M2 and M1 portions of the middle cerebral artery and the internal carotid artery, the aneurysm neck was partially exposed. Most of the aneurysm dome was located beneath the cerebellar tentorium. The posterior communicating artery received

January 1, 2014

accepted after revision

April 22, 2014

published online

June 4, 2014
DOI http://dx.doi.org/

$10.1055 / \mathrm{s}-0034-1378155$. ISSN 2193-6358. (c) 2014 Georg Thieme Verlag KG
Stuttgart · New York

License terms

c) $(1) \$$ 
A
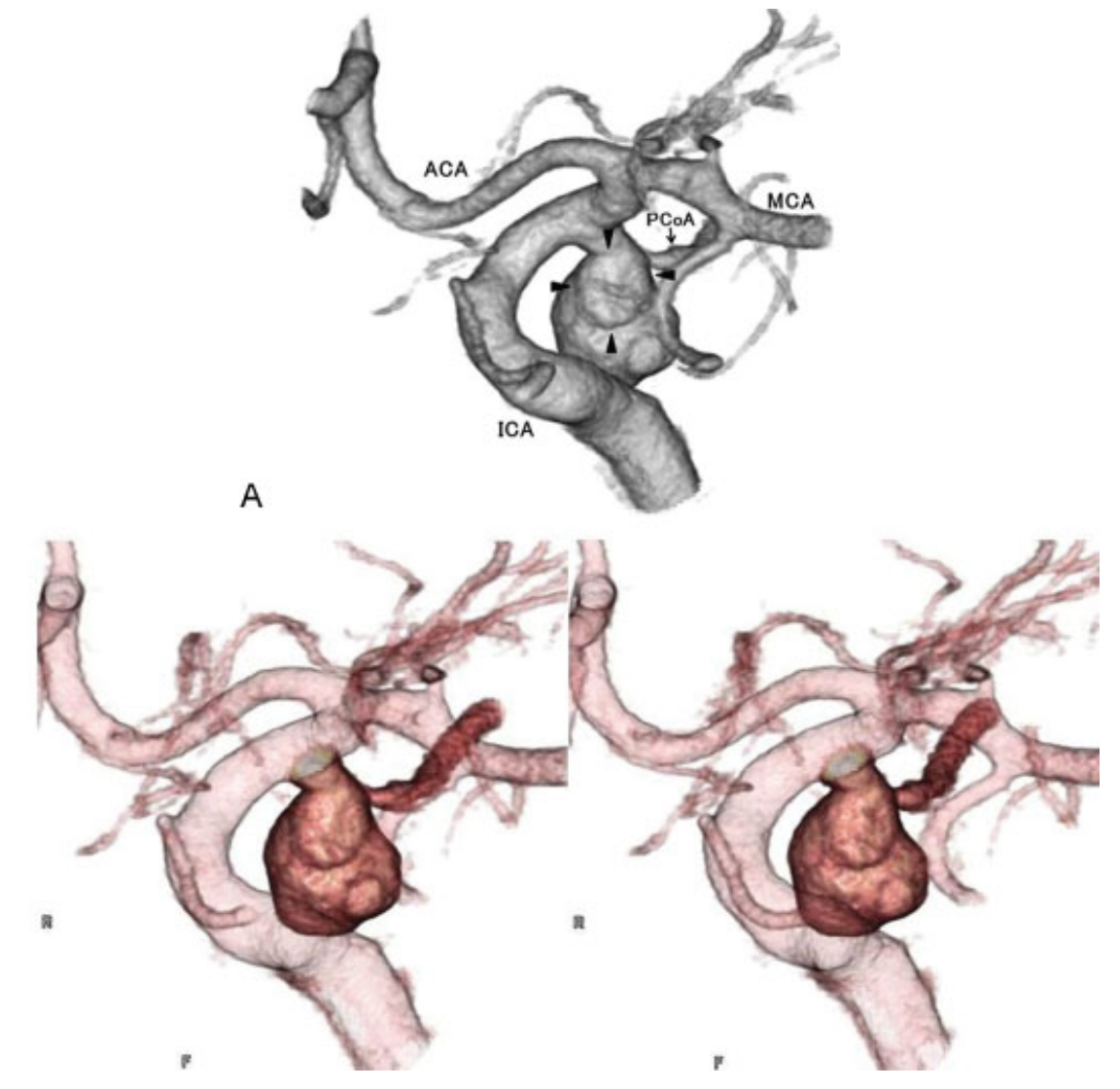

Fig. 1 (A) Volume rendering images of three-dimensional digital subtraction angiography demonstrated a bulging part on the surface of the dome of the left internal carotid-posterior communicating artery aneurysm (arrowheads). ACA, anterior cerebral artery; ICA, internal carotid artery; MCA, middle cerebral artery; PCoA, posterior communicating artery. (B) Stereogram for cross viewing.

originated from the aneurysm neck. It was revealed that the aneurysm dome split the oculomotor nerve. On the surface of the aneurysm dome, a bulging part surrounded by a fenestrated oculomotor nerve and the cerebellar tentorium was noted (-Fig. 2). After the fenestrated oculomotor nerve was dissected from the bulging part with a careful microsurgical technique, neck clipping was performed using two clips (-Fig. 3). After neck clipping, blood flow to the posterior communicating artery was confirmed by Doppler ultrasonography. The post- operative course was uneventful. Although the symptoms of oculomotor nerve paresis did not change just after the operation, they disappeared within 2 weeks.

\section{Discussion}

A fenestrated oculomotor nerve associated with intracranial aneurysm is very rare. To our knowledge, there have been only four case reports. ${ }^{1-4}$ Yasargil first reported a case of IC-PC
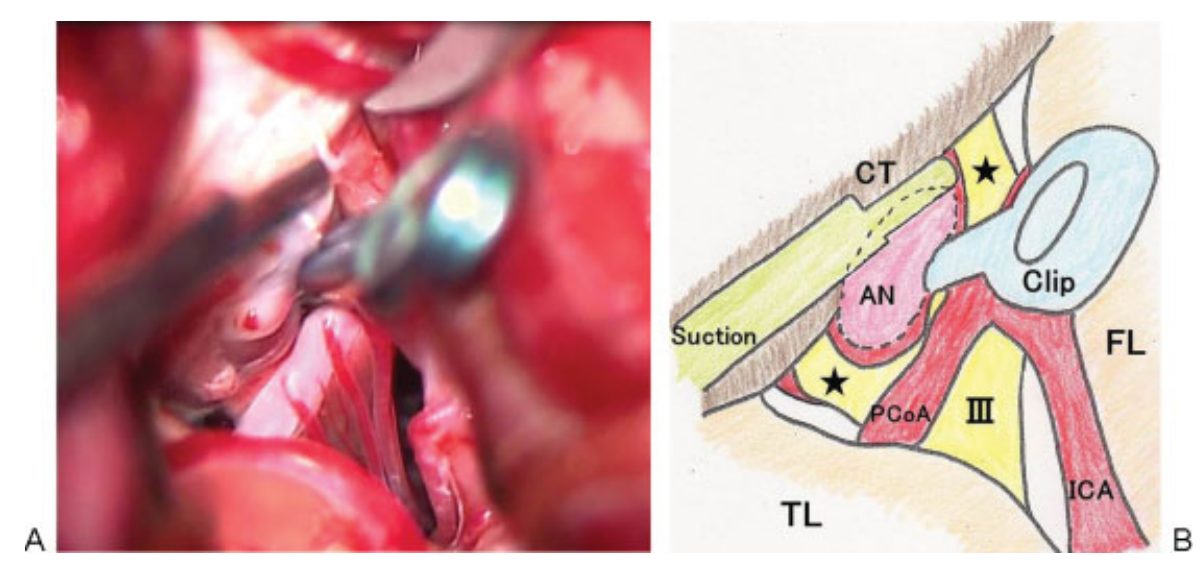

Fig. 2 (A) Operative photograph of left pterional approach. (B) Schematic drawing of left pterional approach. On the surface of the aneurysm dome, a bulging part (dotted line) surrounded by the fenestrated oculomotor nerve (asterisks) and the cerebellar tentorium was noted. III, oculomotor nerve; AN, aneurysm; Clip, applied clip; FL, frontal lobe; ICA, internal carotid artery; PCoA, posterior communicating artery; Suction, suction device; $\mathrm{TL}$, temporal lobe. 


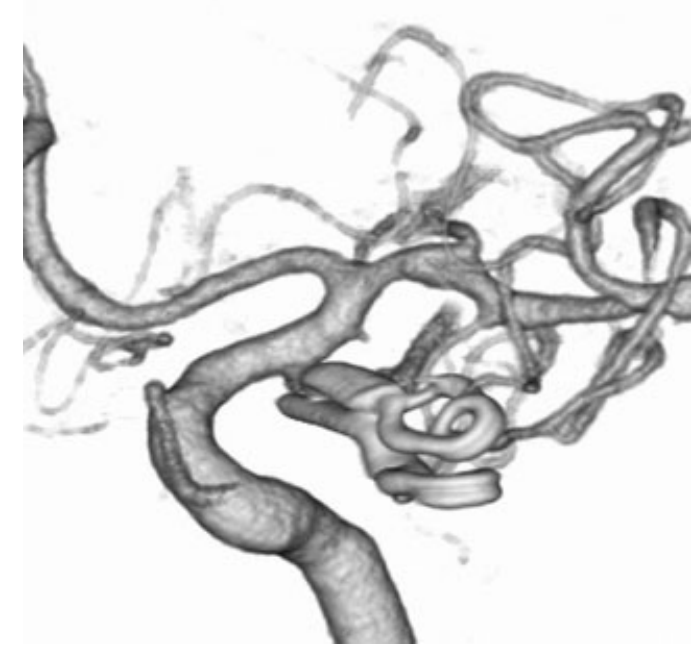

Fig. 3 Post-clipping three-dimensional digital subtraction angiography showing no evidence of residual aneurysm.

aneurysm splitting the oculomotor nerve. ${ }^{4}$ Horiuchi et al also reported a case of IC-PC aneurysm. They concluded that the aneurysm might have ruptured 10 years previously, causing a rent in the nerve into which the aneurysm could grow, as a mechanism of the fenestration of the nerve. ${ }^{2}$ Binning and Couldwell reported the case of a ruptured aneurysm arising from an aberrant duplicated branch of the posterior cerebral artery that split the oculomotor nerve. They believed the artery had traversed the nerve and the aneurysm arose within the region of this fenestration. ${ }^{1}$ Maekawa et al reported a case of unruptured IC-PC aneurysm. They thought that compression by the aneurysm, as it grew, caused fenestration of the nerve. ${ }^{3}$ In the present case, we believed the mechanism was follows. At first, the aneurysm compressed the oculomotor nerve and penetrated it; then it grew beneath the cerebellar tentorium. Finally, the bulging part of it elevated the oculomotor nerve until paresis of it occurred.

None of the four case reports just cited could depict or predict fenestration of the oculomotor nerve before surgery. ${ }^{1-4}$ Even in the present case, we did not predict the formation of fenestration preoperatively; however, we noted a bulging part on the aneurysm dome on volume rendering images of 3D DSA before surgery. In reference to intraoperative findings, we thought the bulging part on the aneurysm dome formed the fenestration of the oculomotor nerve. To our knowledge, this is the first report that 3D DSA depicted a bulging part on an aneurysm that split the oculomotor nerve. We should regard the morphologic features of the aneurysm dome depicted by 3D imaging modalities as very important information for surgical treatment because it may hint at an anatomic relationship between the aneurysm and surrounding structures.

It was reported that coiling of posterior communicating artery aneurysms with oculomotor nerve palsy was associated with high rates of nerve function recovery. ${ }^{5}$ However, Chen et al reported that clipping was associated with a higher probability of complete recovery from oculomotor nerve palsy than coiling. ${ }^{6}$ In the present case, coiling was an alternative treatment option if the patient could not tolerate surgery. But we did not consider coiling as first-line treatment because the posterior communicating artery arose from the neck and the patient was relatively young.

Horiuchi et al reported that they sacrificed the lateral part of the fenestrated oculomotor nerve during surgery, resulting in severe oculomotor nerve palsy. ${ }^{2}$ In contrast, Binning and Couldwell and Maekawa et al reported that oculomotor function was not damaged because they preserved the nerve during surgery. ${ }^{1,3}$ Even in the present case, we thought that careful observation and manipulation of the nerve would enable preservation of oculomotor function. We must keep in mind the possibility of anomaly of the oculomotor nerve, including fenestration, and careful observation and manipulation should be performed to preserve the nerve function when it is suspected during an operation, even though it is very rare.

\section{References}

1 Binning MJ, Couldwell WT. Fenestration of the oculomotor nerve by a duplicated posterior cerebral artery and aneurysm. Case report. J Neurosurg 2009;111(1):84-86

2 Horiuchi T, Kyoshima K, Oya F, Kobayashi S. Fenestrated oculomotor nerve caused by internal carotid-posterior communicating artery aneurysm: case report. Neurosurgery 1997;40(2): 397-398; discussion 398-399

3 Maekawa H, Hadeishi H, Tanaka M. Unruptured internal carotidposterior communicating artery aneurysm splitting the oculomotor nerve: case report. J Stroke 2010;32(2):163-166

4 Yasargil MG. Internal carotid artery aneurysms. In Yasargil MG, ed. Microneurosurgery. Vol 2. Stuttgart, Germany: Thieme; 1984: 76-77

5 Bulsara KR, Jackson D, Galvan GM. Rate of third nerve palsy recovery following endovascular management of cerebral aneurysms. Neurosurg Rev 2007;30(4):307-310; discussion 310-311

6 Chen PR, Amin-Hanjani S, Albuquerque FC, McDougall C, Zabramski JM, Spetzler RF. Outcome of oculomotor nerve palsy from posterior communicating artery aneurysms: comparison of clipping and coiling. Neurosurgery 2006;58(6):1040-1046; discussion 1040-1046 